\title{
Mapeamento de pesquisadores precursores da área de prevenção de drogas em contextos educacionais no Brasil
}

\author{
Mapping precursors researchers of the area of drug prevention in \\ educational contexts in Brazil
}

\section{Mapeo de investigadores precursores del área de prevención de drogas em contextos educacionales en Brasil}

\author{
Julia Ferreira Bernardo ${ }^{1, a}$ \\ juliaf.bernardo@hotmail.com | https://orcid.org/0000-0002-4111-4069
}

Bruno de Oliveira Pinheiro ${ }^{1, b}$

pinheiro.bruno10@hotmail.com | https://orcid.org/0000-0002-3671-3171

Jesús Pascual Mena-Chalco ${ }^{2, c}$

jesus.mena@ufabc.edu.br|https://orcid.org/0000-0001-7509-5532

Denise de Micheli3,d

demicheli@gmail.com | https://orcid.org/o000-0001-8546-4354

\footnotetext{
${ }^{1}$ Universidade Federal de São Paulo. Guarulhos, SP, Brasil.

2 Universidade Federal do ABC. Santo André, SP, Brasil.

${ }^{3}$ Universidade Federal de São Paulo. São Paulo, SP, Brasil.
}

\footnotetext{
a Mestrado em Educação pela Universidade Estadual Paulista.

b Mestrado em Educação e Saúde na Infância e na Adolescência pela Universidade Federal Paulista.

c Doutorado em Ciência da Computação pela Universidade de São Paulo.

${ }^{d}$ Doutorado em Psicobiologia pela Universidade Federal de São Paulo.
}

\section{Resumo}

Apesar do crescente interesse de pesquisadores pela implantação e execução de projetos de prevenção de drogas em contextos educacionais, pouco se conhece sobre a efetividade das ações e projetos desenvolvidos, bem como sobre os pesquisadores que desenvolvem essa temática. Este artigo visou agrupar informações em âmbito nacional dos pesquisadores precursores da área de estudos e conhecimento sobre prevenção de drogas em contextos educacionais e apresentar um método para o mapeamento sistemático para identificação destes pesquisadores. Por meio da análise cientométrica realizada no Catálogo de Teses e Dissertações da CAPES e na Plataforma Lattes, com utilização do software ScriptLattes, foram identificadas as produções bibliográficas, orientações, rede de colaboração e mapa de geolocalização de um conjunto de 61 pesquisadores brasileiros. Conclui-se que estratégias para identificação de pesquisadores e sua produção científica auxiliam na integralização e no compartilhamento de informações, ampliam a troca de conhecimentos e experiências, e evidenciam um mapeamento nacional de pesquisadores de determinadas áreas.

Palavras-chave: Prevenção; Drogas; Educação; Educação em saúde; Bibliometria; Rede social; Pesquisadores. 


\begin{abstract}
Even with the growing interest of researchers in the implementation and execution of drug prevention projects in educational contexts, little is known about the effectiveness of the actions and projects developed, as well as the researchers that develop this theme. This article aimed to group information at the national level of precursors researchers the area of studies and knowledge about drug prevention in educational contexts and to present a method for the systematic mapping that identify these researchers. Using the scientometric analysis in the Bank of Thesis and Dissertations of CAPES and in the Lattes Platform, using the ScriptLattes software, the bibliographic productions, orientations, collaboration network and geolocation map of a group of 61 Brazilian researchers were identified. It is concluded that strategies for the identification of researchers and their scientific production help in the integration and sharing of information, increase the exchange of knowledge and experiences, and evidence a national mapping of researchers in certain areas.
\end{abstract}

Keywords: Prevention; Drugs; Education; Health education; Bibliometry; Social network; Researchers.

\title{
Resumen
}

A pesar del creciente interés de los investigadores acerca de la implantación y ejecución de proyectos de prevención de drogas en contextos educativos, poco se conoce sobre la efectividad de las acciones y proyectos desarrollados, así como sobre los investigadores que desarrollan esa temática. Este artículo buscó agrupar informaciones a nivel nacional de los investigadores precursores del área de estudios y conocimiento sobre la prevención de drogas en contextos educativos y presentar un método para el mapeo sistemático para la identificación de estos investigadores. A través del análisis cientométrico realizado en el Banco de Tesis y Disertaciones de la CAPES y en la Plataforma Lattes, con utilización del software ScriptLattes, se identificaron las producciones bibliográficas, orientaciones, red de colaboración y mapa de geolocalización de un conjunto de 61 investigadores brasileños. Se concluye que estrategias para identificación de investigadores y su producción científica auxilian en la integralización y en el intercambio de informaciones, amplían el intercambio de conocimientos y experiencias, y evidencian un mapeo nacional de investigadores de determinadas áreas.

Palabras clave: Prevención; Drogas; Educación; Educación en salud; Bibliometría; Red social; Los investigadores.

Contribuição dos autores: todos os autores contribuíram significativamente na concepção, desenho do estudo, aquisição, análise, interpretação dos dados, redação do artigo e revisão crítica do conteúdo.

Declaração de conflito de interesses: Este trabalho não apresenta conflito de interesses.

Fontes de financiamento: CAPES, CNPq.

Considerações éticas: Pesquisa documental baseada em dados de domínio público e acesso irrestrito. 0 projeto de doutorado do qual se vincula este estudo foi submetido ao CEP-UNIFESP, via plataforma Brasil, com parecer consubstanciado aprovado n².450.045.

Agradecimento/Contribuições adicionais: não há.

Histórico do artigo: Submetido: 05.abr.2018 | Aceito: 14.maio.2018 | Publicado: 29.jun.2018

Apresentação anterior: não houve.

Licença CC BY-NC atribuição não comercial. Com essa licença é permitido acessar, baixar (download), copiar, imprimir, compartilhar, reutilizar e distribuir os artigos, desde que para uso não comercial e com a citação da fonte, conferindo os devidos créditos de autoria e menção à Reciis. Nesses casos, nenhuma permissão é necessária por parte dos autores ou dos editores. 


\section{Introdução}

O uso, abuso e dependência de substâncias psicotrópicas são temas que, por sua amplitude e impacto em diversas áreas (e.g., saúde, educação, social), tem sido fonte de debates e preocupações no âmbito mundial. A questão perpassa a saúde pública e áreas especializadas, incitando reflexões em diferentes níveis da sociedade e campos do conhecimento na tentativa de compreender o fenômeno e propor estratégias de redução para os problemas deflagrados. Neste contexto, destacam-se os estudos e ações de prevenção e as diversas vertentes relacionadas a estes.

Na última década, inúmeras pesquisas têm retratado uma discussão calorosa a respeito da implantação e execução de projetos de prevenção de drogas em contextos educacionais ${ }^{1-2}$. Isso deve-se ao consumo cada vez mais precoce de substâncias entre os adolescentes, bem como ao consenso de que a escola é um lugar privilegiado para se fazer prevenção, uma vez que é nela que crianças e adolescentes passam grande parte do $\operatorname{dia}^{1-6}$.

Dados do sexto Levantamento sobre o Consumo de Drogas entre Estudantes do Ensino Fundamental e Médio das redes pública e privada de ensino no Brasil, realizado pelo Centro Brasileiro de Informações Sobre Drogas Psicotrópicas (CEBRID) nas 27 capitais brasileiras, demonstraram que 60,5\% dos adolescentes haviam consumido álcool alguma vez na vida, 42,4\% usaram no último ano e 21,1\% usaram no mês anterior à entrevista. A precocidade do início do uso é evidenciada pelo fato de que $15 \%$ dos adolescentes que fizeram uso de drogas ilícitas, $59 \%$ dos que usaram álcool e 9,7\% dos que usaram tabaco tinham entre 10 e 15 anos por ocasião do primeiro uso7.

De fato, a escola é e sempre foi uma importante referência na vida das comunidades, principalmente pelo seu importante papel no desenvolvimento e formação humana. Diferentes estudos na área de prevenção de drogas mostraram que, por estar em contato direto com os adolescentes e apresentar vínculo afetivo com estes, educadores seriam os atores principais para fazerem prevenção ${ }^{4,8}$. A Legislação Brasileira, desde 2006, legitima a necessidade de formação continuada de educadores na área da prevenção de drogas e implantação de projetos pedagógicos com essa finalidade no sistema público e privado de ensino9.

Nesta perspectiva, diferentes pesquisas foram conduzidas avaliando a efetividade de cursos de capacitação sobre prevenção do uso de drogas para educadores ${ }^{10-12}$, adaptação de programas preventivos para utilização em escolas ${ }^{13-14}$ e treinamento de educadores para prevenção do uso de substâncias ${ }^{4}$. No entanto, os resultados evidenciam certa concordância sobre o fato de que, apesar dos esforços dispendidos, os professores permanecem inseguros e temorosos quanto à própria atuação e há certa resistência e despreparo das instituições escolares para trabalhar a temática ${ }^{2,4,10,15}$.

Segundo a literatura, a dificuldade observada em fazer prevenção do uso nocivo de drogas, especialmente no contexto escolar, e/ou replicar modelos de prevenção existentes, deve-se à falta de articulação e conhecimento sobre o que de fato tem sido feito em termos de ações e reconhecimento de seus resultados, bem como a falta de integralidade de informações sobre o tema ${ }^{8,16-17}$. De acordo com pesquisadores do United Nations Office for Drug Control and Crime Prevention ${ }^{18}$ é fundamental que os programas preventivos sejam avaliados e identificadas e disseminadas as ações que se mostrarem efetivas ${ }^{5-6}$.

Mesmo com todo o empenho por parte dos governos e órgãos internacionais que incentivam e reivindicam práticas de prevenção do uso nocivo de drogas, principalmente em contextos educacionais, atualmente relativamente pouco se conhece sobre as ações e projetos desenvolvidos, bem como sobre os pesquisadores que se dedicam a discutir e refletir sobre essa temática. No mesmo sentido, pouco se sabe sobre a quantidade de projetos elaborados, implementados e a efetividade de seus resultados, bem como as características comuns entre os projetos, ações desenvolvidas e diretrizes seguidas.

Diante disso, com o objetivo de identificar e agrupar informações sobre as pesquisas e os pesquisadores que contribuem com estudos sobre o tema, é apresentado um método para identificação e mapeamento sistemático dos pesquisadores precursores, ou seja, pesquisadores seniores que iniciaram os estudos e conhecimentos 
sobre a temática em questão, em âmbito nacional, e formaram pessoas na área. Esta identificação será feita por meio de um estudo cientométrico realizado no Catálogo de Teses e Dissertações da CAPES e na Plataforma Lattes, uma vez que ambos (CAPES e CNPq) aglutinam os mais importantes produtos científicos desenvolvidos no país ${ }^{19}$. A identificação deste grupo pode revelar o começo e a evolução das pesquisas na área de prevenção de drogas em contextos educacionais no país, bem como o impacto delas no panorama nacional de saberes sobre o tema e a rede de colaboração científica composta entre os pesquisadores.

Vale mencionar que vários estudos foram realizados com vistas à revisão de literatura internacional sobre o tema ${ }^{20}$, revisão sistemática sobre programas de prevenção do consumo de álcool dirigido a jovens estudantes brasileiros ${ }^{21} \mathrm{e}$ análise da produção científica sobre programas de prevenção no país ${ }^{22}$. No entanto, até onde se saiba, nenhum estudo foi realizado com objetivo de identificar a rede de colaboração científica que produz conhecimento na área em questão, sendo tal aspecto o diferencial e ponto forte deste estudo.

\section{Método}

O mapeamento dos pesquisadores precursores da área de prevenção de drogas em contextos educacionais foi realizado em quatro etapas descritas na seção de procedimentos abaixo, além disso, foram também utilizados dois programas computacionais operados em diferentes partes do processo de coleta de dados.

\section{Instrumentos}

a) Programa de busca de Teses e Dissertações (T/D) da CAPES: Em 2012, Mena-Chalco e Rocha $^{23}$ analisaram de forma macro todos os trabalhos registrados até 2011 do Catálogo de Teses e Dissertações da CAPES. Como resultado dessa análise, foram elaborados relatórios e armazenados localmente trabalhos defendidos desde 1987 (primeiro ano possível para busca neste repositório) até 2011 (o último ano disponível na época). Os relatórios contêm informações relacionadas com os trabalhos defendidos em instituições brasileiras. As informações coletadas foram: (i) título do trabalho, (ii) autor do trabalho, (iii) orientador, (iv) resumo, (v) palavras-chave, (vi) área do conhecimento e (vii) nome do programa de pósgraduação. O programa que foi desenvolvido para o trabalho de mapeamento de pesquisadores precursores considera como fonte de dados os relatórios do trabalho anteriormente mencionado. O programa elaborado na linguagem de programação Python permite a identificação de trabalhos considerando como palavra de consulta um ou mais termos de busca. A busca é sequencial (cada trabalho é examinado na sua forma sequencial, isto é, elemento a elemento, título, resumo e palavras-chave) e considerada casamento exato de termos baseada em comparação de cadeias (strings). Caso um termo de busca fosse identificado de forma exata no título, no resumo ou nas palavras-chave do trabalho, este seria listado e registrado em uma planilha de dados para análise. No caso das combinações de termos e o uso dos operadores binários AND, a busca foi feita pelo casamento de todos os termos que compõem a combinação. É importante destacar que, em 2012, infelizmente, o Catálogo de Teses e Dissertações da CAPES passou por uma grande reestruturação para adequação à Plataforma Sucupira, e, até o momento, as teses e dissertações defendidas antes de 2013 carecem de informação completa. Para os trabalhos entre 1987 e 2012, as informações importantes para busca não estão presentes, como por exemplo, o catálogo carece de informações de Resumo e palavraschave. O catálogo apresenta apenas uma mensagem indicativa de "Trabalho anterior à Plataforma Sucupira”. Por outro lado, acreditamos que os trabalhos defendidos entre 2012 e 2017, que não estão sendo considerados neste mapeamento de pesquisadores, geraram um impacto menor nos resultados uma vez que nosso objetivo é identificar os pesquisadores precursores (seniores), isto é, pesquisadores pioneiros ou que iniciaram a área de pesquisa de prevenção de drogas em contextos educacionais no Brasil. 
b) Programa de coleta de dados da Plataforma Lattes: ScriptLattes: Refere-se a um software livre, de coleta de dados acadêmicos, projetado e desenvolvido por Mena-Chalco e Cesar-JR ${ }^{24-25}$ para extrair e compilar automaticamente da plataforma lattes dados de um grupo de pesquisadores. Desta forma, o scriptLattes baixa as informações dos currículos Lattes e compila os dados sobre produções bibliográficas, técnicas e artísticas, orientações, projetos de pesquisa, prêmios e títulos, tratando apropriadamente os homônimos, bem como as produções duplicadas e similares. Adicionalmente, a ferramenta permite a criação automática de redes de coautoria entre os membros do grupo e um mapa de geolocalização dos mesmos. Em seguida, são gerados relatórios, com listas de produções e orientações separadas por tipo e colocadas em ordem cronológica invertida. Trata-se de uma ferramenta pioneira na prospecção de extensos conjuntos de dados acadêmicos provenientes da Plataforma Lattes. Esse conhecimento pode ser usado para explorar, identificar ou validar padrões de atividades científicas, trazendo assim informação bibliométrica e/ou cientométrica sobre um grupo ou tema de interesse $\mathrm{e}^{26-27}$.

\section{Procedimentos}

a) Etapa 1- Escolha de termos e combinações como estratégia de busca: Para selecionar os termos associados às teses e dissertações sobre o tema em questão, foi necessário um levantamento amplo, com buscas em artigos, teses, dissertações, descritores em ciências da saúde - DeCS, da base de dados BIREME, entre outros meios que pudessem indicar as palavras mais utilizadas. Além disso, contamos com o auxílio de pesquisadores experientes na área, os quais puderam ampliar e aprimorar a escolha dos termos mais significativos. Participaram desta etapa seis pessoas convidadas, três pós-graduandos do departamento de educação e saúde na infância e adolescência da UNIFESP, campus Guarulhos, e três profissionais da área de drogas e prevenção. A importância de contar com o apoio de outros pesquisadores é demonstrada em vários tipos de estudo, como as pesquisas de revisão sistemática e meta-análise, por exemplo. Nestes casos, além do pesquisador principal, são incluídos outros revisores que possuam conhecimento aprofundado no tema de estudo e assuntos adjacentes. Isto garante maior qualidade e cientificidade ao trabalho desenvolvido ${ }^{28-29}$. Esse apoio foi solicitado em outros momentos desta pesquisa com objetivo de encontrar caminhos mais adequados para cada fase do projeto.

A fim de não perder nenhum dado relevante, nesta primeira etapa foram considerados o maior número de termos possíveis e realizadas combinações com o operador lógico AND que se julgaram importantes para estratégia de busca, dado que a área em discussão se apresenta de forma multidisciplinar, uma vez que, quando se fala em prevenção de drogas no contexto educacional, várias áreas estão presentes como, por exemplo, saúde, educação, sociologia, antropologia. Na segunda etapa, filtram-se os termos e/ou combinações que foram relevantes para o rastreamento dos trabalhos específicos do tema abordado. A rodagem pode acontecer várias vezes, até se chegar aos termos e/ou combinações mais significativas, que comporão a lista final de busca.

Neste estudo foram necessárias duas rodagens para estabelecer a lista definitiva, a primeira considerando 179 termos e/ou combinações e a segunda, após análise e estudo de cada um e sua relevância de busca, considerando 78 combinações, apresentadas na íntegra na Figura 1 dos resultados. Deste modo, foram utilizadas somente combinações de ao menos duas palavras como estratégia de busca definitiva, tendo em vista que não havia palavras isoladas nos termos finais escolhidos para identificar as teses e dissertações mais importantes, pois estas rastreavam pesquisas que não interessavam ao estudo.

b) Etapa 2 - Definição de critérios de inclusão/exclusão: Estabelecer critérios de inclusão/ exclusão consiste em escolher, numericamente e estatisticamente, um ponto para designar uma condição. Esta escolha depende do tipo de estudo e do objetivo do trabalho. Nesta fase definimos os critérios empiricamente, dado que não há, previamente, limiares de corte estabelecidos para este tipo de pesquisa. 
Tendo em vista o número de teses e dissertações encontradas, foi importante que se fizesse um estudo para verificação da fidedignidade dos termos escolhidos, por meio da frequência de ocorrência de termos por T/D e estabelecidos critérios de inclusão/exclusão. Percebeu-se que, quanto mais ocorrências de pelo menos uma das combinações de termos escolhidas como estratégia de busca nas teses e dissertações, mais específicas da área de prevenção de drogas no contexto educacional elas eram. Após uma análise apurada de cada ocorrência de combinação de termos e sua representatividade nos trabalhos encontrados, foi estabelecido o limiar de corte de pelo menos 3 ocorrências por teses e dissertações, da lista final com 78 combinações de termos. Assim, foram selecionadas para a terceira fase somente as teses e dissertações que continham ao menos três termos diferentes na busca por título, resumo e palavras chaves, e excluídas aquelas que continham menos de três.

c) Etapa 3: Seleção dos orientadores: Encontradas as teses e dissertações, já é possível identificar todos os pesquisadores que orientaram tais trabalhos. Embora o programa possibilitasse identificar os alunos de mestrado e doutorado, autores das teses e dissertações, o objetivo deste estudo concentrou-se em identificar os professores orientadores, para mapear os pesquisadores precursores, com nível de senioridade na área de interesse, que iniciaram os estudos sobre o tema no país, difundiram conhecimento e formaram pessoas. Além disso, foi estabelecido outro critério de inclusão/exclusão, um filtro com limiar de corte de ao menos duas orientações, para designar maior coerência entre aqueles que de fato podem ser considerados da área de prevenção de drogas em contextos educacionais e aqueles que somente fizeram uma orientação sobre o tema, uma vez que esta área caminha em conjunto com outras áreas do conhecimento.

d) Etapa 4: Coleta de informações acadêmicas: A coleta de dados acadêmicos registrados na Plataforma Lattes foi realizada por meio do scriptLattes. Com o nome de cada um do grupo de pesquisadores, encontrados na etapa anterior, e seus respectivos currículos Lattes, foram extraídas as informações de produção acadêmica de seus currículos. Desta forma, com o relatório gerado, são obtidos os resultados referentes ao panorama nacional relevante contendo informações deste grupo dos últimos 29 anos (1987 a 2016).

\section{Resultados}

Serão apresentados os dados obtidos com a rodagem do programa que consulta os repositórios institucionais da CAPES (Catálogo de Teses e Dissertações), em que foi possível identificar as teses e dissertações defendidas entre os anos de 1987 a 2011, relacionadas ao tema em relevância, bem como selecionar os orientadores desses trabalhos, pesquisadores precursores, os quais fizeram parte da segunda análise. Em seguida, serão apresentados os dados referentes ao relatório gerado pelo scriptLattes, em que se obteve a compilação automática de produções bibliográficas, orientações, projetos de pesquisa, rede de coautoria, e mapa de geolocalização dos pesquisadores selecionados na primeira parte.

A Figura 1 apresenta o conjunto de combinações utilizadas como estratégia de busca selecionadas para a identificação de teses e dissertações no catálogo de trabalhos da CAPES, totalizando 78 combinações. 
abuso de drogas AND educacao AND prevencao; abuso de drogas AND prevencao AND adolescente*; abuso de drogas AND prevencao AND escola*; acoes de prevencao AND drogas AND escola*; adolescencia AND drogas AND escola*; adolescencia AND drogas AND prevençao; adolescente* AND drogas AND escola*; adolescente* AND drogas AND prevencao; alcool AND prevencao AND adolescencia; alcool AND prevencao AND escola; ambiente escolar AND drogas AND prevencao; uso abusivo de drogas AND prevencao AND escola*; autonomia AND drogas AND educacao; autonomia AND drogas AND prevencao AND escola*; avaliacao de programa* AND drogas AND escola*; capacitacao AND educador* AND drogas; capacitacao AND professor* AND drogas; competencia social AND drogas AND prevencao; corpo docente AND capacitacao AND drogas; corpo docente AND formacao AND drogas; crianca AND drogas AND prevencao; desenvolvimento social AND drogas AND prevencao; docente* AND drogas AND prevencao; drogas de abuso AND prevencao AND escola; drogas ilicitas AND prevencao AND escola; educacao AND drogas; educacao AND drogas AND projeto de prevencao; educacao AND prevencao AND drogas; educacao continuada AND drogas AND escola*; educacao continuada AND professores AND drogas; educacao em saude AND drogas; educacao para a saude AND drogas AND prevencao; educacao preventiva AND drogas AND escola*; educador* AND capacitacao AND drogas; educador* AND drogas AND prevencao; ensino AND drogas AND prevencao; escola* AND drogas AND prevencao; estudantes AND drogas AND prevencao; familia AND drogas AND prevencao; familia AND escola AND drogas; fatores de protecao AND educacao AND drogas AND prevencao; fatores de risco AND educacao AND prevencao AND drogas; formacao continuada AND drogas AND prevencao; formacao continuada AND professores AND prevencao; formacao de educadores AND drogas; habito* AND drogas AND prevencao; jovem AND drogas AND prevencao; jovens AND drogas AND prevencao; juventude AND drogas AND prevencao; maconha AND adolescencia AND prevencao; medida* AND preventiva* AND drogas AND escola*; overdose AND adolescencia AND drogas AND prevencao; pais AND escola AND drogas; politica* AND social* AND drogas AND prevencao; politica* AND publica* AND prevencao AND drogas; politica* AND publica* AND prevencao AND escola*; praticas pedagogicas AND drogas AND prevencao; prevencao AND drogas AND escola AND adolescente*; prevencao de drogas AND escola*; prevencao na escola AND drogas; prevencao primaria AND drogas AND escola*; prevencao universal AND drogas AND escola*; professor* AND drogas AND prevencao; programas de treinamento AND drogas AND educacao; projeto de prevencao AND drogas AND educacao; promocao da saude AND drogas AND escola*; reducao de dano* AND drogas AND escola*; reducao de dano* AND escola*; reducao de dano* AND escola* AND prevencao; saude escolar AND drogas; uso indevido de drogas AND prevencao AND educacao; uso indevido de drogas AND prevencao AND escola*; uso nocivo de drogas AND prevencao; vulnerabilidade* AND drogas AND prevencao; vulnerabilidade* AND drogas AND educacao; vulnerabilidade* AND drogas AND escola*; vulnerabilidade* AND social AND drogas AND escola*; vulnerabilidade* AND social AND drogas AND prevencao.

Figura 1 - Combinações de Termos Utilizados para Busca no Catálogo de Teses e Dissertações da Capes. Fonte: Os autores (2018).

Foram examinados 607.389 documentos disponíveis no Catálogo de Teses e Dissertações da CAPES dos anos de 1987 a 2011, entre teses, dissertações acadêmicas e profissionalizantes. A partir das 78 combinações de palavras como estratégias de busca apresentadas acima, foram identificadas 1453 teses e/ou dissertações que continham ao menos 1 das 78 combinações em seus títulos, resumos e/ou palavras-chaves. Aplicado o critério de inclusão/exclusão de 3 ocorrências diferentes por trabalho, chegou-se ao número final de 450 trabalhos. A Figura 2 (a) mostra a ocorrência de combinações por quantidade de teses e dissertações, já a Figura 2 (b), a proporção de teses e dissertações por ano (isto é, o número de trabalhos identificados dividido pelo número de trabalhos defendidos no Brasil entre os anos 1987 e 2011).

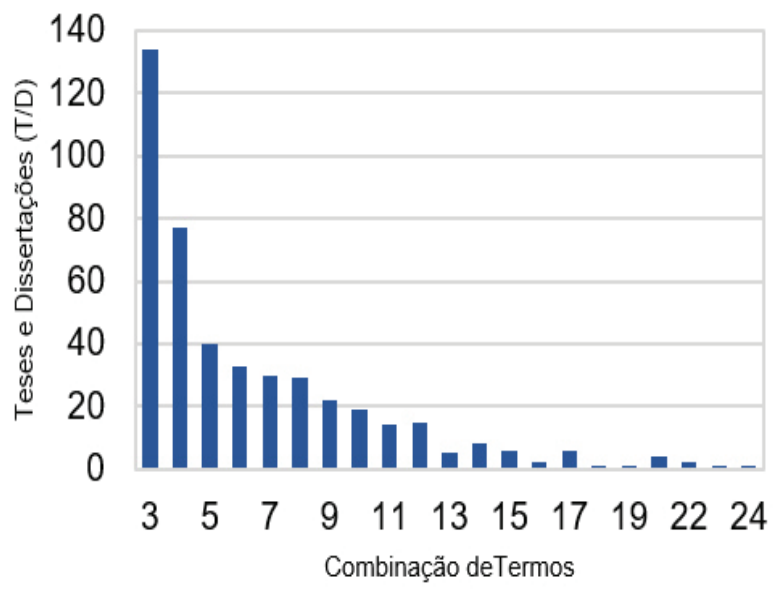

(a)

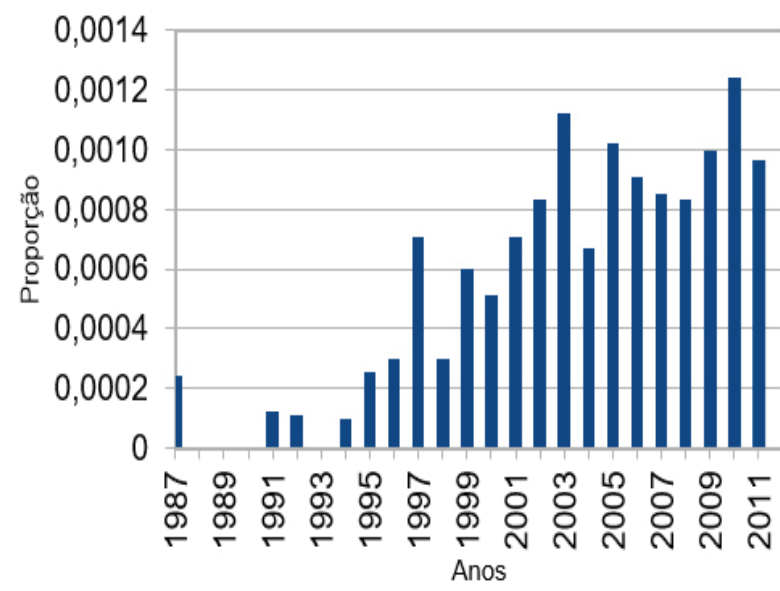

(b)

Figura 2 - Ocorrência de (a) Combinações de Termos por Teses e Dissertações e (b) Proporção de Trabalhos por Ano. Fonte: Os autores (2018). 
Sobre a frequência de combinação de termos, o trabalho que apresentou maior ocorrência teve 24 combinações diferentes associadas. Contudo, a maior parte das teses e dissertações teve entre 3 e 8 ocorrências associadas, representando 51,79\% do total, sendo a ocorrência de 3 combinações a sua grande maioria, 134 trabalhos.

$\mathrm{Na}$ amostra identificada de 450 trabalhos, a estratégia de busca "educacao AND drogas" resultou em 226 trabalhos diferentes, seguido de "escola AND drogas AND prevencao", presente em 208 trabalhos, "adolescente* AND drogas AND escola*" em 191, "adolescente* AND drogas AND prevencao" em 154 e, "educacao AND prevencao AND drogas", aparecendo em 147 trabalhos. Somente 5 estratégias de buscai, das 78, apareceram apenas em 1 trabalho, e outras $4^{\text {ii }}$ foram encontrados em 2 trabalhos distintos.

$\mathrm{O}$ restante apareceu mais de 3 vezes em trabalhos diferentes, sendo que a grande maioria, $64 \%$ aproximadamente, apareceu em mais de 10 trabalhos diferentes.

Sobre a data da defesa, conforme mostra a Figura 2(b), observa-se que a taxa de crescimento de trabalhos na área de pesquisas sobre prevenção de drogas em contextos educacionais vem aumentando de forma sistemática (proporção crescente) nos últimos anos, sendo superior ao número total de trabalhos, entre teses e/ou dissertações, defendidos no período.

A relação normalizada entre os trabalhos da área em estudo e os trabalhos totais encontrados no repositório da CAPES mostra uma proporção crescente, demonstrada pelo aumento das taxas de crescimento em relação ao período. Entre os anos de 2009 e 2011, houve uma maior eclosão de trabalhos defendidos sobre o tema, somando 153 trabalhos, dos 450 encontrados.

Nesta amostra foram identificados 86 tipos diferentes de instituições de ensino de vários lugares do Brasil, a saber: universidades, faculdades, campis, centros de pesquisas e educação, e fundações de Ensino. A Tabela 1 mostra a quantidade de trabalhos e de instituições de ensino considerando a região do país.

Tabela 1 - Teses e Dissertações e Locais por Região

\begin{tabular}{lcc}
\hline Região & Teses e dissertações & Inst. de Ensino \\
\hline Centro-Oeste & 32 & 8 \\
\hline Nordeste & 68 & 15 \\
\hline Norte & 4 & 3 \\
\hline Sudeste & 268 & 42 \\
\hline Sul & 78 & 18 \\
\hline Total Geral & 450 & 86 \\
\hline
\end{tabular}

Fonte: Os autores (2018).

A instituição de ensino que mais contribuiu com pesquisas na área de prevenção de drogas em contextos educacionais na região sudeste foi a Universidade de São Paulo (USP), campus Ribeirão Preto, com 41 T/D, seguida pela Universidade de São Paulo - campus São Paulo com 23 T/D, Universidade Federal de São Paulo (UNIFESP) com 20 T/D e Pontifícia Universidade Católica de São Paulo (PUC-SP) com 19 T/D. Em outras regiões do país, as instituições de ensino que mais contribuíram foram: na região sul, a Universidade Federal do Rio Grande do Sul, com 15 T/D; na região centro-oeste, a Universidade de Brasília, com 14 T/D; na região nordeste, a Universidade Federal do Ceará, com 13 T/D e, na região norte, a Universidade Federal do Amazonas, com 2 trabalhos dos 450.

\footnotetext{
i prevencao universal AND drogas AND escola*; drogas de abuso AND prevencao AND escola; programas de treinamento AND drogas AND educacao; uso nocivo de drogas AND prevencao; overdose AND adolescencia AND drogas AND prevencao.

ii formacao continuada AND professores AND prevencao; corpo docente AND formacao AND drogas; prevencao primaria AND drogas AND escola*; corpo docente AND capacitacao AND drogas.
} 
Embora a USP - Ribeirão Preto tenha sido a universidade com maior número de trabalhos defendidos no tema no período de 1987 e 2011, ela corresponde somente a 9,11\% do total de 450 trabalhos. Isto porque há, nesta amostra, uma difusão dos trabalhos correlatos à prevenção de drogas em contextos educacionais dentre as 86 instituições de ensino encontradas.

Quanto ao número de orientadores, identificou-se 376 docentes que orientaram algum dos 450 trabalhos mapeados, podendo ser o orientador principal e/ou coorientador. A partir da utilização do critério de inclusão de pesquisadores com no mínimo duas orientações sobre o tema, foi obtido o resultado de 61 docentes. Estes pesquisadores foram designados por este estudo como pesquisadores precursores, ou seniores da área, os quais foram submetidos à coleta de dados usando o scriptLattes para compilar informações gerais contidas na Plataforma Lattes, bem como informações de publicações e colaboração entre este grupo.

O Quadro 1 mostra todos os pesquisadores precursores mapeados por meio do método proposto, identificados por siglas correspondentes aos seus nomes e sobrenomes; a área de atuação principal de cada um, definida por critério constituído a partir de suas publicações, grupos de pesquisa, projetos e resumo, identificadas pelas siglas S (saúde), E (educação), ES (educação e saúde), esta última subdividida em $+\mathrm{S}$ (mais saúde do que educação), +E (mais educação do que saúde), M (multidisciplinar); título de doutorado, instituição de trabalho e região do país, também identificadas por siglas: SE (Sudeste), S (Sul), NE (Nordeste), N (Norte) e CO (Centro-Oeste).

Quadro 1 - Pesquisadores Precursores da Área de Prevenção de Drogas em Contextos Educacionais

\begin{tabular}{|c|c|c|c|c|}
\hline Nome & Área & Título & Instituição & Região \\
\hline B.W.F. & E & Educação & PUC/ RS & $S$ \\
\hline E.C.P. & $E$ & Ciências Sociais & UFMT & $\mathrm{CO}$ \\
\hline F.B. & $E$ & Educação & UNESP/ Bauru & SE \\
\hline H.R.C. & $E$ & Educação & UFRN & NE \\
\hline R.A.M. & $E$ & Psicologia & UNESP/ Marilia & SE \\
\hline V.M.N.S.P. & $E$ & Educação & PUC/ São Paulo & SE \\
\hline A.A.L. & $E S(+E)$ & Educação & UFPR & $S$ \\
\hline A.V.G.M. & ES (M) & Educação & UFSCAR & SE \\
\hline D.M. & $E S(+S)$ & Psicobiologia & UNIFESP & SE \\
\hline F.K.C. & $E S(+S)$ & Neurologia & UNESP/ Botucatu & SE \\
\hline L.C.A.W. & ES (M) & Psicologia & UFSCAR & SE \\
\hline M.F.O.S. & ES (M) & Psicologia & UNB & $\mathrm{CO}$ \\
\hline M.G.C.F. & $E S(+S)$ & Enfermagem & USP/ Ribeirão Preto & SE \\
\hline M.L.M.A. & ES (M) & Educação & UMA & SE \\
\hline M.M.R. & $\mathrm{ES}(+\mathrm{S})$ & Saúde Mental & UMESP & SE \\
\hline M.M.S. & $\mathrm{ES}(+\mathrm{S})$ & Ciências Fisiológicas & UFES & SE \\
\hline P.N.C.P. & $E S(+S)$ & Enfermagem & UFC & NE \\
\hline S.H.K. & $E S(+S)$ & Educação & UFRS & $S$ \\
\hline S.M.V.B. & ES (M) & Educação & USP/ Ribeirão Preto & SE \\
\hline S.R.G.P. & ES (M) & Psicologia Clínica & UNESP/ Marília & SE \\
\hline A.A.W.S. & $S$ & Psicologia & UFPB & $\mathrm{NE}$ \\
\hline
\end{tabular}


(continua)

\begin{tabular}{|c|c|c|c|c|}
\hline Nome & Área & Título & Instituição & Região \\
\hline A.F.C.J. & $S$ & Odontologia Preventiva e social & UFPE & NE \\
\hline A.K.B.P. & $S$ & Enfermagem & UFC & NE \\
\hline A.M.P.C. & $S$ & Psicologia Escolar e DH & USP/ Ribeirão Preto & SE \\
\hline A.T. & $S$ & Psicologia Clínica & PUC/ Campinas & SE \\
\hline B.V.C. & $S$ & Psicologia Social & UFSC & $S$ \\
\hline C.S.H. & $S$ & Psicologia & UFRS & $S$ \\
\hline D.R.G.C.A. & $S$ & Ciências Médicas & ULBRA & $S$ \\
\hline D.X.S.F. & $S$ & Psiquiatria & UNIFESP & SE \\
\hline E.C.S.G.D. & $S$ & Enfermagem Psiquiátrica & USP/ Ribeirão Preto & SE \\
\hline E.H. & $S$ & Psicologia Clínica & USP & SE \\
\hline E.J. & $S$ & Ciências políticas e Sociologia & Ministério da Saúde & $\mathrm{CO}$ \\
\hline F.P. & $S$ & Ciências Médicas & UFRS & $S$ \\
\hline I.A.H.P. & $S$ & Imunologia & $\mathrm{PUC} / \mathrm{GO}$ & $\mathrm{CO}$ \\
\hline I.D. & $S$ & Fisiopatologia & UNESP/ Botucatu & SE \\
\hline J.A.L. & $S$ & Saúde Pública & UFMG & SE \\
\hline J.U.B. & $S$ & Ciências Médicas & ULBRA & S \\
\hline L.G.P.S. & $S$ & Clínica Obstétrica & Sec.Mun. Saúde RJ & SE \\
\hline L.M.F.P. & $S$ & Enfermagem & UFC & $\mathrm{NE}$ \\
\hline L.S.P. & $S$ & Psiquiatria & ULBRA & $S$ \\
\hline M.A.S. & $S$ & Psicologia Clínica & USP & SE \\
\hline M.A.V.L. & $S$ & Enfermagem & USP/ Ribeirão Preto & SE \\
\hline M.C.O.C. & $S$ & Ciências aplicadas a Pediatria & UEFS & NE \\
\hline M.C.S.M. & $S$ & Psicologia & UFES & SE \\
\hline M.D.B.C. & $S$ & Enfermagem & UEM & $S$ \\
\hline M.H.V.B.C. & $S$ & Antropologia & PUC/ São Paulo & SE \\
\hline M.L.F.O. & $S$ & Saúde Coletiva & UEM & $S$ \\
\hline M.L.O.S.F. & $S$ & Farmacologia & UNIFESP & SE \\
\hline M.M. & $S$ & Enfermagem & UFG & $\mathrm{CO}$ \\
\hline M.P.L.C. & $S$ & Psicologia Clínica & UFPB & NE \\
\hline M.S.O. & $S$ & Psiquiatria & PUC/RS & $S$ \\
\hline M.S.P. & $S$ & Enfermagem & UFBA & NE \\
\hline M.V.G.C. & $S$ & Pediatria & FCM Santa Casa & SE \\
\hline R.R.L. & $S$ & Psiquiatria & UNIFESP & SE \\
\hline S.B.A. & $S$ & Psiquiatria & UNIFESP & SE \\
\hline S.C.P. & $S$ & Psiquiatria & USP/ São Paulo & SE \\
\hline S.R.T. & $S$ & Saúde da Criança e do Adolescente & UERJ & SE \\
\hline T.M.A. & $S$ & Medicina e Saúde & UFBA & NE \\
\hline
\end{tabular}




\begin{tabular}{ccccc} 
Nome & Área & Título & Instituição & Região \\
\hline T.M.R. & S & Psicobiologia & UFJF & SE \\
\hline V.L.A.R.A. & S & Psicologia Escolar e DH & PUC/ Campinas & SE \\
\hline V.S.F.P. & S & Psicologia Social & USP & SE \\
\hline
\end{tabular}

Fonte: Os autores (2018).

Os dados obtidos mostraram que este grupo está distribuído em 15 estados brasileiros, atuam em 34 instituições diferentes, sendo 32 relacionados a Campis diferentes da mesma Universidade, Universidades diferentes, Centros Universitários e Faculdades, e 2 setores de serviços públicos. As instituições quemais concentram esses pesquisadores sãoa Universidade de São Paulo, campus Ribeirão Preto e a Universidade Federal de São Paulo, ambas com 5 representantes cada. São em sua maioria mulheres ( $\mathrm{n}=42)$ e, em relação a titulação acadêmica, há maior prevalência de pesquisadores titulados na área da psicologia ( $\mathrm{n}=15)$, seguido pela educação $(n=9)$, enfermagem $(\mathrm{n}=9)$ epsiquiatria $(\mathrm{n}=5)$, o restante são titulados em diferentes áreas, grande parte em ciências da saúde.

Sobre a área de atuação, nota-se que há um maior número atuando principalmente na área da saúde, com 41 pesquisadores. A área de interface educação e saúde teve 14 pesquisadores sendo: educação e saúde (mais saúde do que educação), educação e saúde (mais educação do que saúde) e educação e saúde (multidisciplinar) e, na área da educação, 6 representantes. A definição das subáreas da educação e saúde se deu por meio da avaliação das informações contidas nos currículos de cada pesquisador onde foi possível identificar atuações distintas, mesmo dentro desta interface. Já os pesquisadores definidos em educação ou saúde são aqueles que se dedicam mais dentro de cada uma dessas áreas, embora tenham dialogado uma ou mais vezes com outros campos do conhecimento. Neste sentido, vale lembrar que um dos objetivos deste estudo foi mapear os pesquisadores precursores da área de prevenção de drogas em contextos educacionais, que por si, denota a interação entre diferentes áreas do conhecimento.

Em relação à localização geográfica dos pesquisadores, estes concentraram-se principalmente na região Sudeste, com 34 pesquisadores, seguida da região Sul com 12 pesquisadores, região Nordeste, com 10 pesquisadores e região Centro-Oeste do Brasil com 5 (Figura 3).

Esses achados evidenciam a concentração de pesquisadores na região Sudeste do país, em contraponto com nenhuma representatividade na região Norte.
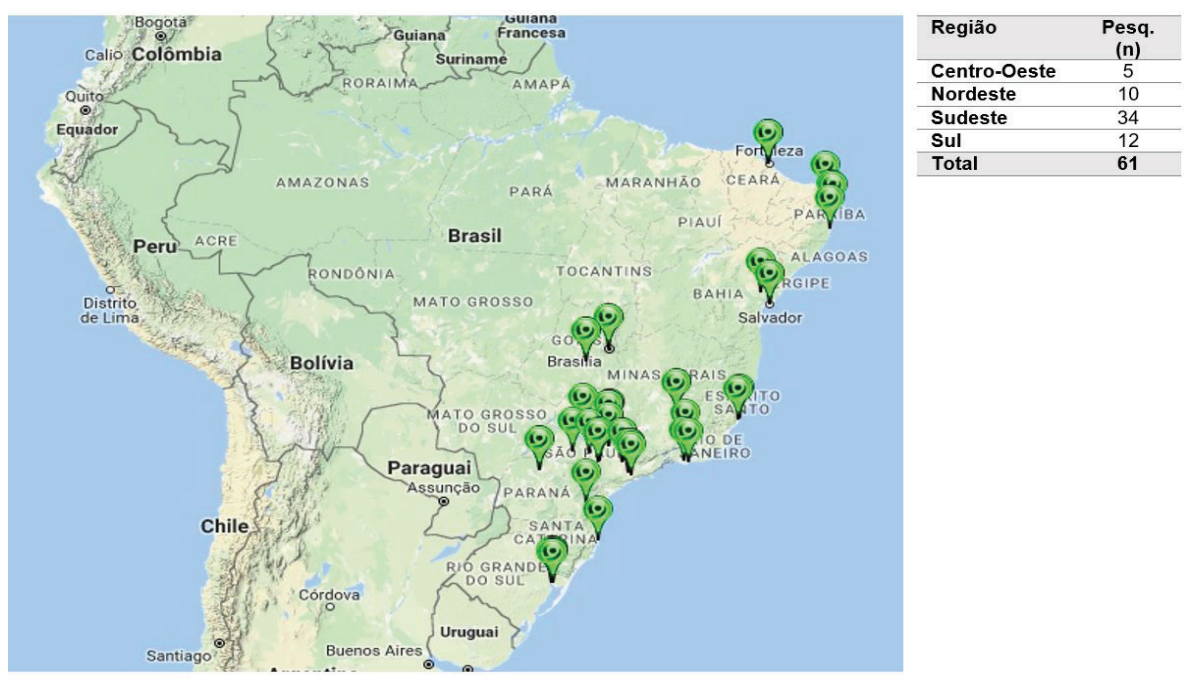

Figura 3 - Mapa de Geolocalização dos Pesquisadores.

Fonte: Os autores (2018). 
A Figura 4 apresenta a rede de colaboração entre os pesquisadores identificados, considerando as diferentes áreas de atuação. Cada ponto está relacionado a um pesquisador, e as arestas representam a colaboração entre eles. A espessura de cada aresta é proporcional ao número de colaborações entre dois pesquisadores. Assim, arestas mais grossas caracterizam alto grau de colaboração, enquanto as mais finas, menor grau. Observa-se que a maioria dos pesquisadores encontrados neste mapeamento colaborou com um ou mais pesquisadores, $\mathrm{e}$ há poucos pontos isolados. Ou seja, somente 14 dos 61 pesquisadores não tiveram colaboração com nenhuma pessoa desta rede, o que revela que aproximadamente $78 \%$ deles tinham algum grau de colaboração dentro do grupo. Além disso, nota-se maior interação entre os pesquisadores da área da saúde e estes com a área da educação e saúde, em suas subáreas - mais saúde do que educação e multidisciplinar. Os pontos menores em azul escuro, que representam a área de educação, estão mais afastados do centro, e somente um deles está imerso na maior trama desta rede, colaborando com pesquisadores das outras áreas.

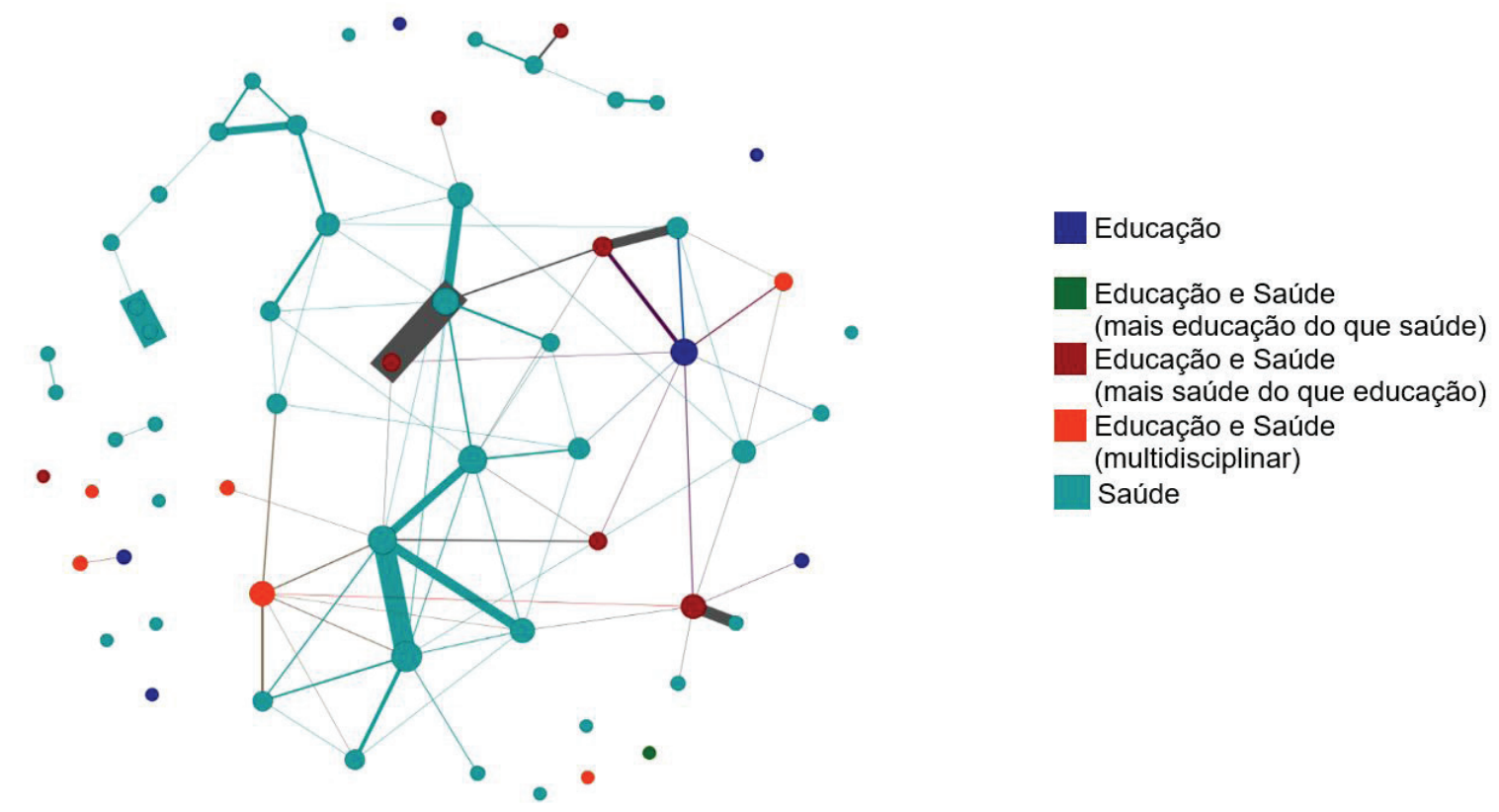

Figura 4 - Rede de colaboração entre os 61 pesquisadores Fonte: Os autores (2018).

Em relação à produção do grupo identificado, a Tabela 2 revela a quantidade total dos diferentes tipos de publicação, orientações e projetos, desde o início dos seus registros no currículo Lattes até agosto de 2017.

Tabela 2 - Quantidade Total de Publicações, Orientações e Projetos dos Pesquisadores.

\begin{tabular}{|lc|}
\hline Produção Bibliográfica & \\
\hline Artigos completos publicados em periódicos & 5196 \\
\hline Livros publicados/organizados ou edições & 502 \\
\hline Capítulos de livros publicados & 1847 \\
\hline Textos em jornais de notícias/revistas & 796 \\
\hline Trabalhos completos publicados em anais de congressos & 1315 \\
\hline Resumos expandidos publicados em anais de congressos & 591 \\
\hline Resumos publicados em anais de congressos & 8961 \\
\hline Demais tipos de produção bibliográfica & 415 \\
\hline Total & 19623 \\
\hline
\end{tabular}




\begin{tabular}{|lc|}
\hline Orientações ou supervisões & \\
\hline Orientações em andamento & 173 \\
\hline Tese de doutorado & 121 \\
\hline Dissertação de mestrado & 29 \\
\hline Trabalho de conclusão de curso de graduação & 75 \\
\hline Iniciação científica & 5 \\
\hline Orientações de outra natureza & \\
\hline Total & $\mathbf{4 3 6}$ \\
\hline Supervisão e Orientações concluídas & 63 \\
\hline Supervisão de pós-doutorado & 692 \\
\hline Tese de doutorado & 1544 \\
\hline Dissertação de mestrado & 541 \\
\hline Monografia de conclusão de curso de aperfeiçoamento/especialização & 791 \\
\hline Trabalho de conclusão de curso de graduação & 1245 \\
\hline Iniciação científica & 697 \\
\hline Orientações de outra natureza & \\
\hline Total & $\mathbf{5 5 7 3}$ \\
\hline Projetos de pesquisa & 850 \\
\hline Total de projetos de pesquisa & \\
\hline
\end{tabular}

Fonte: Os autores (2018).

As produções elencadas na Tabela 2 se referem a toda produção do grupo, independentemente do tema ou campo do conhecimento em que desenvolveram, ou desenvolvem, estudos. Isso quer dizer que nem tudo que está quantificado é relativo ao conhecimento científico da área de prevenção de drogas em contextos educacionais. Embora os pesquisadores precursores mapeados colaborem com a área, também é comum realizarem pesquisas em outros campos do conhecimento, uma vez que muitos deles trabalham com outros temas de relevância para saúde e/ou educação.

\section{Discussão}

Este estudo teve por objetivo principal identificar, em âmbito nacional, os pesquisadores que contribuem com estudos sobre prevenção de drogas no contexto educacional. Para realização deste mapeamento, desenvolveu-se um método específico, paralelamente ao uso da ferramenta denominada scriptLattes, previamente utilizada em outros estudos para compilação de dados acadêmicos ${ }^{24-25,30}$.

Considerando o mapeamento realizado, observou-se maior prevalência de pesquisadores da área da saúde na produção de estudos relativos à prevenção de drogas no contexto educacional, ainda que tais estratégias preventivas sejam realizadas dentro de espaços da educação, como escolas. Mesmo com incentivos governamentais para que os educadores se aproximem e sejam capacitados a lidar com esta temática, observa-se ainda uma carência desta classe profissional ao se discutir o assunto.

Em uma pesquisa de revisão de literatura, sobre prevenção de drogas em escolas, desenvolvida por CarliniCotrin \& Pinsky ${ }^{20}$, verificou-se que não havia no Brasil, até aquela época, produções científicas nacionais, tampouco tradição acadêmica que pudesse dialogar com o âmbito internacional, em relação à prevenção do abuso de drogas na escola. Tais produções, segundo as autoras, estavam concentradas na área do Direito e na 
Medicina. Embora avanços de pesquisas e aumento de produções na área tenham sido demonstrados neste artigo pela Figura 2b, no que diz respeito ao crescimento de teses e dissertações defendidas sobre o tema em relação ao total, entre os anos de 1987 e 2011, e em relação à área de atuação dos pesquisadores, ainda há predominância da saúde, o que corrobora, de certa maneira, com o estudo mencionado, do ano de 1989.

$\mathrm{O}$ avanço das tecnologias da informação e comunicação auxiliou diversos campos do conhecimento, permitindo maior acessibilidade sobre a produção científica. Essas tecnologias, incorporadas à necessidade de agregar informações sobre um tema, possibilitam estabelecer indicadores da atividade científica, bem como, identificar estratégias, ações e todo conhecimento consolidado sobre um assunto. Análises bibliométricas e cientométricas, por exemplo, são amplamente utilizadas para quantificar a produção, disseminação e uso da informação, e quantificar a ciência como disciplina ou atividade, respectivamente. Deste modo, é possível reunir produções acadêmicas de um assunto de interesse ${ }^{31}$. Isto acontece em pesquisas de revisão sistemática, levantamento de publicações dentro de uma ou mais bases de dados, em periódicos, catálogo de teses e dissertações, sobre uma área ou um assunto específico.

Haeffner e Guimarães ${ }^{32}$ realizaram um levantamento sobre a produção científica nacional e internacional na área de neurociência e comportamento dentro da base Web of Science. O estudo mostrou um avanço deste tipo de pesquisas no Brasil e no mundo de 1981 a 2014. Já a pesquisa de Martins ${ }^{33}$, fez uma análise bibliométrica para identificação de estudos sobre o Zica Vírus em cinco bases de dados científicas diferentes. A autora evidenciou a falta de estudos nacionais na área e a necessidade de investimentos em pesquisas neste tema.

Em 2006 foi realizada uma revisão sistemática para identificação de programas de prevenção de álcool para jovens estudantes ${ }^{21}$. A revisão encontrou somente sete programas consistentes, espalhados em países diferentes. Os autores exploraram os aspectos metodológicos de cada programa e seus resultados, bem como evidenciaram a escassez destes projetos e a necessidade de maior dedicação sobre o assunto.

Alguns métodos também são desenvolvidos para finalidades cientométricas e bibliométricas, como uma proposta metodológica que possibilitou a investigação da produção científica no campo da saúde coletiva no periódico Cadernos de Saúde Pública no ano de 2011. Este método, apoiado na teoria de análise de redes sociais, analisa redes semânticas de título dos artigos e coautoria dentro do periódico. Com esta estratégia, foi possível identificar os temas mais evidentes nas publicações do ano de 2011 e a rede de coautoria relacionada ${ }^{34}$.

A análise da produção científica nacional sobre programas de prevenção do consumo de drogas entre os anos de 1991 e 2001, feito por Canoletti e Soares ${ }^{22}$, possibilitou a identificação de 28 trabalhos diferentes, projetos desenvolvidos e publicados no Brasil. Além da categorização das abordagens utilizadas, o referido estudo demonstrou que a maioria dos projetos se encontra na região Sudeste do Brasil, em específico no eixo Rio-São Paulo. Estes dados são similares à localização da rede de pesquisadores precursores mapeada por este estudo, revelada na Figura 3, bem como sobre a defesa de teses e dissertações sobre o assunto, Tabela 1, ambas concentradas no Sudeste. Tais achados estão relacionados com a concentração de Instituições de Ensino Superior (IES) por região do Brasil. Dados do CENSO sobre educação superior no Brasil de 2013 mostram que, em relação ao número de IES, a região Sudeste compreende aproximadamente $48 \%$ do total nacional, com 1145 IES, seguida da região Nordeste e Sul, com 18\% e 17\% respectivamente, a região CentroOeste com 10\%, e a região Norte com apenas 6\% (146 IES) ${ }^{35}$.

Estudos sobre redes de pesquisadores de determinada área têm sido produzidos com o fim de identificação de colaboração entre eles e divulgação científica de determinado assunto. Sobral, Silva, Bufrem e Coêlho ${ }^{30}$ realizaram um estudo sobre a rede de pesquisa colaborativa na área da saúde tropical em uma universidade do país, fazendo referência à análise de redes sociais na perspectiva da colaboração científica. O estudo utilizou o scriptLattes para o levantamento da produção do grupo e criou um banco de dados bibliométricos com as compilações dos currículos de cada pesquisador. A análise dos resultados mostrou interação entre a maioria dos pesquisadores, por se tratar de uma rede endógena, dentro de um mesmo programa de pós-graduação. 
No que tange à rede de colaboração encontrada nesta pesquisa, a maioria dos pesquisadores identificados está integrada na trama da rede. Este dado segue a tendência de crescimento dos trabalhos em colaboração. $\mathrm{Na}$ área da saúde, por exemplo, os artigos em colaboração entre os anos de 2004 e 2006 representavam 80\%, diferentemente do que acontecia entre os anos de 1998 e 2000, em que a publicação de autoria única era a maioria, aproximadamente $60 \%$ dos artigos ${ }^{36}$.

Um estudo cientométrico e da rede de colaboração de pessoas, pesquisadores precursores, na área de prevenção de drogas e educação pode ampliar o conhecimento em relação ao tema e identificar os pesquisadores que contribuíram e impactaram a área tanto na produção de pesquisas quanto na formação de pessoas, além de possibilitar o agrupamento de informações sobre as estratégias, vertentes teóricas e projetos práticos que têm sido consolidados no Brasil nas últimas décadas. Apesar do programa scriptLattes ter realizado a compilação das produções bibliográficas da rede de pesquisadores encontrada, a qualificação e a categorização desta produção não fazem parte do escopo deste artigo. O mapeamento dos pesquisadores foi realizado de forma sistemática, oportunizando diferentes análises a partir dos resultados encontrados.

Embora o método tenha se mostrado adequado para os objetivos propostos, sabe-se que há outros pesquisadores que se empenham em discutir e estudar questões relacionadas à prevenção de drogas e educação e que não foram identificados neste mapeamento, uma vez que o catálogo de teses e dissertações da CAPES agrega as informações somente entre 1987 e 2011. Entretanto, outros estudos serão realizados para encontrar novos pesquisadores, destacados após o ano de 2011, utilizando-se desses dados iniciais, a partir da expansão da rede por crescimento, por intermédio dos orientandos desses pesquisadores, bem como por coautoria. Outro ponto a ser ressaltado é que não se pode afirmar que todos os pesquisadores identificados são estudiosos especificamente do tema escolhido, isto porque este tema perpassa outras áreas e campos do conhecimento. $\mathrm{O}$ que se reconhece é que tais pesquisadores contribuíram com estudos correlatos à área de prevenção de drogas em contextos educacionais e fazem parte do panorama nacional sobre o tema.

\section{Conclusões}

O método apresentado foi considerado válido em sua proposta, mapeou pesquisadores precursores de uma área ou tema de interesse e possibilitou compilar as informações contidas nos currículos Lattes em um relatório único, quantificando as produções bibliográficas, orientações e projetos e conhecendo a rede de colaboração entre os pesquisadores e sua localização geográfica dentro do território nacional. Os pesquisadores encontrados, da área de prevenção de drogas em contextos educacionais, foram identificados com critérios definidos de forma intuitiva e evolutiva em todo processo de criação do método, obedecendo as possibilidades tanto das bases de dados escolhidas quanto dos programas utilizados. Em relação à plataforma da CAPES, os dados sobre teses e dissertações estão disponíveis somente até 2011. Neste cenário, escolheu-se selecionar os orientadores desses trabalhos que tiveram no mínimo duas orientações até este ano, demonstrando um nível de senioridade na área de interesse.

Mesmo que o catálogo de dados escolhido limite uma busca após o ano de 2011, isto não interferiu no objetivo do estudo que foi o de identificar pesquisadores precursores da área em âmbito nacional. $\mathrm{O}$ grupo identificado, com 61 pesquisadores, não se remete a todos os pesquisadores da área, e sim de um grupo mais antigo, com nível de senioridade, que se reconhece a importância de suas contribuições para o campo de conhecimento sobre prevenção de drogas em contextos educacionais, bem como na formação (orientação) de pessoas na área.

A criação e utilização de estratégias para identificação de pesquisadores e sua produção científica é uma possibilidade de auxiliar a integralização e o compartilhamento de informações, ampliar a troca de conhecimento e experiências, e assim evidenciar uma rede de colaboração científica de um tema de interesse. 


\section{Referências}

1. Sodelli M. Uso de drogas e prevenção: da desconstrução da postura proibicionista às ações redutoras de vulnerabilidade. São Paulo: Iglu; 2010.

2. Moreira A, Vóvio CL, Micheli D. Drug abuse prevention in school: challenges and possibilities for the role of the educator. Educ Pesqui [Internet]. 2015 [citado em 2018 jun. 06];41(1):119-134. doi: http:// dx.doi.org/10.1590/S1517-97022015011670

3. Souza FB, Andrade ALM, Rodrigues TP, Nascimento MO, Micheli D. Avaliação das concepções de educadores de escolas públicas e particulares sobre uso de drogas: um estudo exploratório. Estud Pesqui Psicol [Internet]. 2015 [citado em 2018 jun. 06];15(3):1081-95. Disponível em: https://goo.gl/qvHqMf

4. Nascimento MO, Micheli D. Evaluation of different school-based preventive interventions for reducing the use of psychotropic substances among students: a randomized study. Ciênc Saúde Coletiva [Internet]. 2015 [citado em 2018 jun. 06];20(8):2499-510. doi: http://dx.doi.org/10.1590/1413$\underline{81232015208.15152014}$

5. Foxcroft DR, Tsertsvadze A. Universal alcohol misuse prevention programmes for children and adolescents: Cochrane sys- tematic reviews. Perspect Public Health [Internet]. 2012 [citado em 2018 jun. 06];132(3):128-34. doi: http://journals.sagepub.com/doi/pdf/10.1177/1757913912443487

6. Strøm HK, Adolfsen F, Fossum S, Kaiser S, Martinussen M. Effectiveness of school-based preventive interventions on adolescent alcohol use: a meta-analysis of randomized controlled trials. Subst Abuse Treat Prev Policy [Internet]. 2014 [citado em 2018 jun. 06];9:48. doi: https://doi.org/10.1186/1747-597X-9-48

7. Carlini EA, Noto AR, Sanchez SV, Carlini C, Locatelli DP, Abeid L, et al. VI Levantamento Nacional Sobre o Consumo de Drogas Psicotrópicas Entre Estudantes do Ensino Fundamental e Médio das Redes Pública e Privada de Ensino nas 27 Capitais Brasileiras. Brasília: CEBRID; 2010.

8. Pereira APD, Paes AT, Sanchez ZM. Factors associated with the implementation of programs for drug abuse prevention in schools. Rev Saude Publica [Internet]. 2016 [citado em 2018 jun. 07];50:44. doi: http://dx.doi.org/10.1590/S1518-8787.2016050005819

9. Presidência da República (BR). Casa Civil. Subchefia de Assuntos Jurídicos. Lei no 11.343, de 23 de Agosto de 2006. Institui o Sistema Nacional de Políticas Públicas sobre Drogas - Sisnad. DOU [Internet]. 2006 ago. 24 [citado em 2018 jun. 07]. Disponível em: https://goo.gl/22fijo

10. Araldi JC, Njaine K, Oliveira MC, Ghizoni AC. Representações sociais de professores sobre o uso abusivo de álcool e outras drogas na adolescência: repercussões nas ações de prevenção na escola. Interface (Botucatu) [Internet]. 2012 [citado em 2018 jun. 07];16(40):135-48. Disponível em: http://dx.doi. org/10.1590/S1414-32832012005000002

11. Monteiro EP, Gomide HP, Silveira PS, Ronzani TM. Curso de prevenção ao uso de drogas: Descrição e avaliação de satisfação. Estud Psicol (Natal) [Internet]. 2016 [citado em 2018 jun. 07];21(3):328-36. doi: http://dx.doi.org/10.5935/1678-4669.20160031

12. Lopes JM. Avaliação do processo de implementação de programa de prevenção escolar do uso de drogas na percepção dos professores participantes [tese]. Florianópolis: Programa de Pós-Graduação em Psicologia, Universidade Federal de Santa Catarina; 2016.

13. Sanchez ZM, Sanudo A, Andreoni S, Schneider DR, Pereira APD, Faggiano F. Efficacy evaluation of the school program unplugged for drug use prevention among Brazilian adolescents. BMC Public Health [Internet]; 2016;16:1206. Disponível em: https://goo.gl/e95qjW

14. Sanchez ZM, Valente JY, Sanudo A, Pereira APD, Cruz JI, Schneider D, et al. The \#Tamojunto Drug Prevention Program in Brazilian Schools: a Randomized Controlled Trial. Prev Sci. 2017;18(7):772-82. doi: $\underline{\text { https://doi.org/10.1007/s11121-017-0770-8 }}$

15. Cordeiro ILS, Silva DMA, Vecchia, MA. A escola diante do aluno que faz uso de álcool e drogas: O que dizem os professores? Pesqui prát psicossociais [Internet]. 2016 [citado em 2018 jun. 07];11(2):356-68. Disponível em: http://pepsic.bvsalud.org/pdf/ppp/v11n2/07.pdf

16. Babor TF, Caetano R, Casswell S, Edwards G, Giesbrecht N, Graham K. Alcohol: No Ordinary Commodity. Research and Public Policy. 2 ed. New York: Oxford University Press; 2010.

17. Cahill HW. Challenges in adopting avidence-based school drug education programmes. Drug and Alcohol Review [Internet]. 2007 [citado em 2018 jun. 07];26(6):673-9. doi: https://doi. org/10.1080/09595230701613593 
18. United Nations Office for Drug Control and Crime. Prevention Lessons learned in drug abuse prevention: a global review. Nova Iorque; 2002.

19. Stumpf IRC. Disponibilização de teses e dissertações em comunicação em texto completo: projeto de pesquisa. In: $24^{\circ}$ Congresso Brasileiro de Ciências da Comunicação; 2001 set. 3-7; Campo Grande: Intercom; 2001.

20. Carlini-Cotrim B., Pinsky I. Prevenção ao abuso de drogas na escola: uma revisão da literatura internacional recente. Cad Pesq [Internet].1989 [citado em 2018 jun. 07];(69):48-52.

21. Barroso T, Barbosa A, Mendes A. Programas de prevenção do consumo de álcool em jovens estudantes: revisão sistemática. Rev Referencia [Internet]. 2006 [citado em 2018 jun. 07];2(3):33-44. Disponível em: https://goo.gl/jGscWD

22. Canoletti B, Soares CB. Programas de Prevenção ao Consumo de Drogas no Brasil: uma análise da produção científica de 1991 a 2001. Interface (Botucatu) [Internet]. 2005 [citado em 2018 jun. 07];9(16):115-29. doi: http://dx.doi.org/10.1590/S1414-32832005000100010

23. Mena-Chalco JP, Rocha V. Caracterização do banco de teses e dissertações da CAPES. In: $4^{\circ}$ Encontro Brasileiro de Bibliometria e Cientometria; 2001 maio 14-16; Recife; 2014.

24. Mena-Chalco JP, Cesar Junior RM. ScriptLattes: an open-source knowledge extraction system from the lattes platform. J Braz Comp Soc [Internet]. 2009 [citado em 2018 jun. 07];15(4):31-9. doi: http:// dx.doi.org/10.1007/BF03194511

25. Mena-Chalco JP, Cesar-Junior RM. Prospecção de dados acadêmicos de currículos Lattes através do scriptLattes. In: Hayashi MCPI, Leta J, organizadores. Bibliometria e Cientometria: reflexões teóricas e interfaces. São Carlos: Pedro \& João Editores; 2013. p.109-28.

26. Nicholson, S. The basis for bibliomining: frameworks for bringing together usage- based data mining and bibliometrics through data warehousing in digital library services. Informations Processing and Management [Internet]. 2006 [citado em 2018 jun. 07];42(3):785-804.

27. Peng F, Mccallum A. Information extraction from research papers using conditional random fields. Information Processing and Management [Internet]. 2006 [citado em 2018 jun. 07]; 42(4):963-79. doi: https://doi.org/10.1016/j.ipm.2005.09.002

28. Sampaio RFE, Mancini MC. Estudos de Revisão Sistemática: um Guia Para Síntese Criteriosa da Evidência Científica. Rev bras fisioter [Internet]. 2007 [citado em 2018 jun. 07];11(1):83-9. doi: http:// dx.doi.org/10.1590/S1413-35552007000100013

29. De-La-Torre-Ugarte-Guanilo MC, Takahashi RF, Bertolozzi, MR. Revisão sistemática: noções gerais. Rev Esc Enferm [Internet]. 2011 [citado em 2018 jun. 07];45(5). doi: http://dx.doi.org/10.1590/S0080$\underline{62342011000500033}$

30. Sobral NV, Silva FM, Bufrem LS, Coêlho MRCD. Produção científica colaborativa na área da saúde tropical: uma análise da rede de colaboração do Programa de Pós-Graduação em Medicina Tropical da Universidade Federal de Pernambuco. RECIIS, Rev. Eletronica Comun., Inf. Inov. Saude [Internet]. 2016 [citado em 2018 jun. 07];10(1). Disponível em: https://goo.gl/Md1QDv

31. Macias-Chapula CA. O papel da informetria e da cienciometria e sua perspectiva nacional e internacional. Ci Inf [Internet]. 1998 [citado em 2018 jun. 07];27(2):134-40. doi: http://dx.doi.org/10.1590/S010019651998000200005

32. Haeffner C, Guimarães JA. Produção científica na área de neurociência e comportamento indexada na base de dados Web of Science. RECIIS, Rev. Eletronica Comun., Inf. Inov. Saude [Internet]. 2016 [citado em 2018 jun. 07];10(3). Disponível em: https://goo.gl/4idwrS

33. Martins MFM. Análise bibliométrica de artigos científicos sobre o vírus Zika. In: RECIIS - Rev. Eletron. Comum. Inf. Inov. Saúde [Internet]. 2016 [citado em 2018 jun. 07]:10(1). Disponível em: https://goo.gl/NbA7o6

34. Rodrigues AAAO, Fadigas IS, Rosa MG, Ferreira APC, Souza ES, Pereira HBB. Um método para analisar a temática de periódicos voltados para a saúde Coletiva. RECIIS, Rev. Eletronica Comun., Inf. Inov. Saude [Internet]. 2017 [citado em 2018 jun. 07];11(1). Disponível em: https://goo.gl/uz9Fcj

35. INEP. Censo da educação superior 2013: resumo técnico [Internet]. Brasília; 2015 [citado em 2018 jun. 07]. Disponível em: http://inepdata.inep.gov.br.

36. Viacava F. Produção científica dos cursos de pós-graduação em Saúde Coletiva no período 1998-2006. Ciência \& Saúde Coletiva [Internet].2010 [citado em 2018 jun. 07];15(4):1977-88. doi: http://dx.doi. org/10.1590/S1413-81232010000400013 